\title{
BIOMECHANICAL EVALUATION OF TWO DIFFERENT VERTEBRAL INTERBODY DEVICES BY USING QCT-BASED CASE-SPECIFIC NONLINEAR FINITE ELEMENT MODELS: A PRELIMINARY REPORT
}

Péter Varga ${ }^{1}$, Péter $\mathrm{Nédli}^{2}$, Tibor Csákány $^{3}$, Márta Kurutzné Kovács ${ }^{2}$, Péter Pál Varga ${ }^{3}$ ${ }^{1}$ Julius Wolff Institute and Berlin-Brandenburg School for Regenerative Therapies, Charitè Universitätsmedizin Berlin, Germany

${ }^{2}$ Department of Structural Mechanics, Budapest University of Technology and Economics, Hungary

${ }^{3}$ National Center for Spinal Disorders, Budapest, Hungary

peter.varga@charite.de

\begin{abstract}
Interbody devices are widely used to replace the degenerated discs of the spine. For this purpose, a novel methodology uses cement instead of conventional spacers, which is hypothesized to provide smoother transition of forces, lower risk of bone tissue damage, thus smaller subsidence, reduced risk of further pathological deformations and other complications. This new treatment approach has been compared with the conventional method experimentally by mechanical loading of human vertebral motion segments treated with either of these. The present study aimed at complementing the that work with finite element analysis and, by performing in silico mechanical testing of QCT-based case specific models incorporating the elasto-plastic behavior of bone, providing better understanding of experimental results, in particular, the differences between the two sample groups equipped with the different spacer types. This report presents the applied numerical methodology as well as the first results, which are in line with the experimental ones. Besides providing deeper insight into the experimental outcomes, these models are expected to provide a basis for virtual parameter analysis studies, which may help to optimize the surgical procedure.
\end{abstract}

Keywords: vertebral spine, intervertabral disc degeneration, interbody device, motion segment, QCT, finite element analysis

\section{Introduction}

In severe pathological cases of degenerated intervertebral discs surgical intervention is required to restore the original distance of the adjacent vertebrae and stabilize the spine. The most often applied solution is the fusion of adjacent vertebrae using graft material, which is in most cases combined with the application of interbody implants like metal or PEEK cages and spacers to provide mechanical stability and preserve the restored height. This approach leads to reduced range of motion but offers increased global stability of the spine and relieve low back pain. In the severe cases when the pathology of the intervertebral disc is accompanied by impaired bone competence e.g. in osteoporosis, application of the standard fixation approaches using interbody implants can be inadequate due to the reduced bone quality and the degeneration of the vertebral endplates and may lead to damage of the bone tissue at the interface via excessive subsidence of the spacer, yielding to further complications, pain, instability and potentially to the collapse of the 
motion segment. ${ }^{1-4}$ A novel method aims at circumventing the drawbacks of the conventional techniques by using cement spacers. In the frame of this approach bone cement is injected into the gap of the removed parts of the degenerated intervertebral disc and the remaining space is filled with graft material to foster fusion of the adjacent vertebrae. ${ }^{5-6}$ The cement spacer accurately follows the actual contours of the endplates, provides larger contact surface and thus smoother transition of forces along the bone-cement interface. This technique is therefore believed to reduce stress concentrations and therefore the risk of bone tissue damage and extensive spacer subsidence leading to pathological deformations.

An in vitro experimental loading setup has recently been developed and utilized to obtain and compare the apparent mechanical properties of human spinal motion segments fixed with either a conventional or the cement spacer. Even though the mechanical testing represents the gold standard in assessing these properties and provides the ground truth, not all details can be obtained during the experiments and the investigation of all key influencing factors requires a large number of specimens. In silico approaches offer an alternative tool which can be efficiently used to complement or even replace experiments. Finite element models can be utilized to mimic the mechanical conditions and provide insight into the details of stress and strain fields in space and time. Such models may therefore help to better understand and correctly interpret the experimental results, ${ }^{7}$ however, have first to be carefully validated against the real tests. Once validated, the models can be used to investigate the influence of certain factors like changes in the material properties or adjustment of the applied boundary conditions.

The goal of this study was to investigate and compare, by means of finite element analysis, the loading and damage profile of human motion segments stabilized with conventional and with cement spacers. In particular, we aimed at (i) generating case-specific finite element models based on CT images, (ii) validating these against the experimental results, (iii) using the details provided by the validated numerical analyses to better characterize and thus more accurately understand the observed differences between the two operation techniques and (iv) performing a sensitivity analysis to identify and optimize the major factors influencing the results to provide hints for enhancement of the surgical procedures. In this manuscript we report the preliminary results of steps (i-iii).

\section{Methods}

Sixteen thoracic and lumbar spine motion segments, i.e. two adjacent vertebrae with the intervertebral disc were extracted from eight human cadaver spines. Donors were four female and four male with age ranging from 52 ys to 88 ys $(70.6 \pm 13.9 \mathrm{ys})$. All experiments were in line with the Hungarian legislative requirements and the ethical approval was granted by the Ethical Commission of the Semmelweis University, Budapest, Hungary. The samples were cleaned of surrounding soft tissues and stored frozen at $-20^{\circ} \mathrm{C}$ until further processing. The superior and inferior parts of the segments were shallowly embedded in polymer plastic. The thickness of the embedding layers was closely $10 \mathrm{~mm}$, but varied slightly due to the different sizes of the vertebrae and the fact that the volume of the resin was standardized. As the X-ray attenuation coefficient of the selected embedding resin was close to that of water, small glass beads were placed into the resin shortly before polymerization in order to mark the upper and lower surfaces of both embedding layers. Three to four beads were used for each surface. The planes of the inferior and 
posterior resin layers were closely, but not completely parallel. A prepared and embedded specimen is shown in Figure 1.

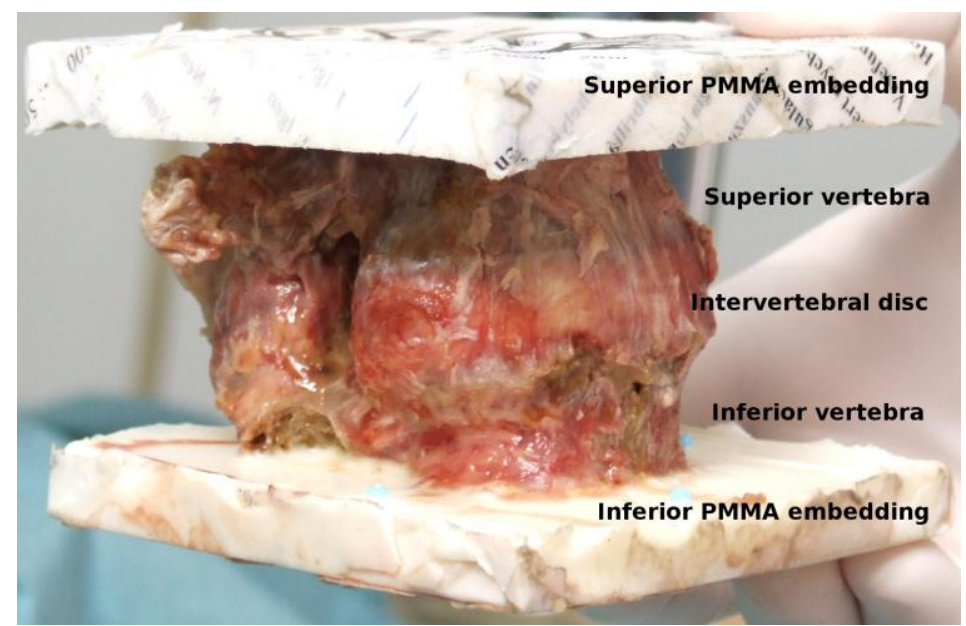

Figure 1. Human spinal motion segment specimen prepared for mechanical testing. The blue dots in the embedding layers are the glass beads.

Quantitative computed tomography (QCT) images of the motion segments were assessed by means of a conventional clinical instrument (Hitachi Presto, Hitachi Medical Corporation, Tokyo, Japan) according to the lumbar spine acquisition protocol. Scanning settings were $120 \mathrm{kVp}$ energy, $150 \mathrm{~mA}$ current, $150 \mathrm{~ms}$ exposure time, 512x512 pixel matrix, $0.47 \mathrm{~mm}$ in-plane pixel size, $0.75 \mathrm{~mm}$ slice thickness. The samples were scanned in native state submerged in a water filled box to mimic the attenuation and scattering effects of soft tissues surrounding the vertebral spine in vivo.

Subsequently, the motion segments were operated with either a conventional method using a polyether ether ketone (PEEK) spacer, or the novel technique using polymethylmethacrylate (PMMA) cement. The motion segments were assigned randomly to the both groups. The posterior segments of the vertebrae were cropped on one side, a small window was cut on the annulus of the intervertebral disc and the nucleus removed. In case of the conventional method a commercial PEEK spacer was placed between the adjacent vertebral endplates. In the other case, PMMA cement was injected into the space left opened by the removed disc parts. To separate and elucidate the effect of bone quality, no posterior fixation instrumentation have been used. The graft material used in in vivo cases to fill the remaining gaps was not used here. Following the operation, the CT scans were repeated with the same settings described above. The CT images acquired before and after the cement injection process are shown in Figure 2. 

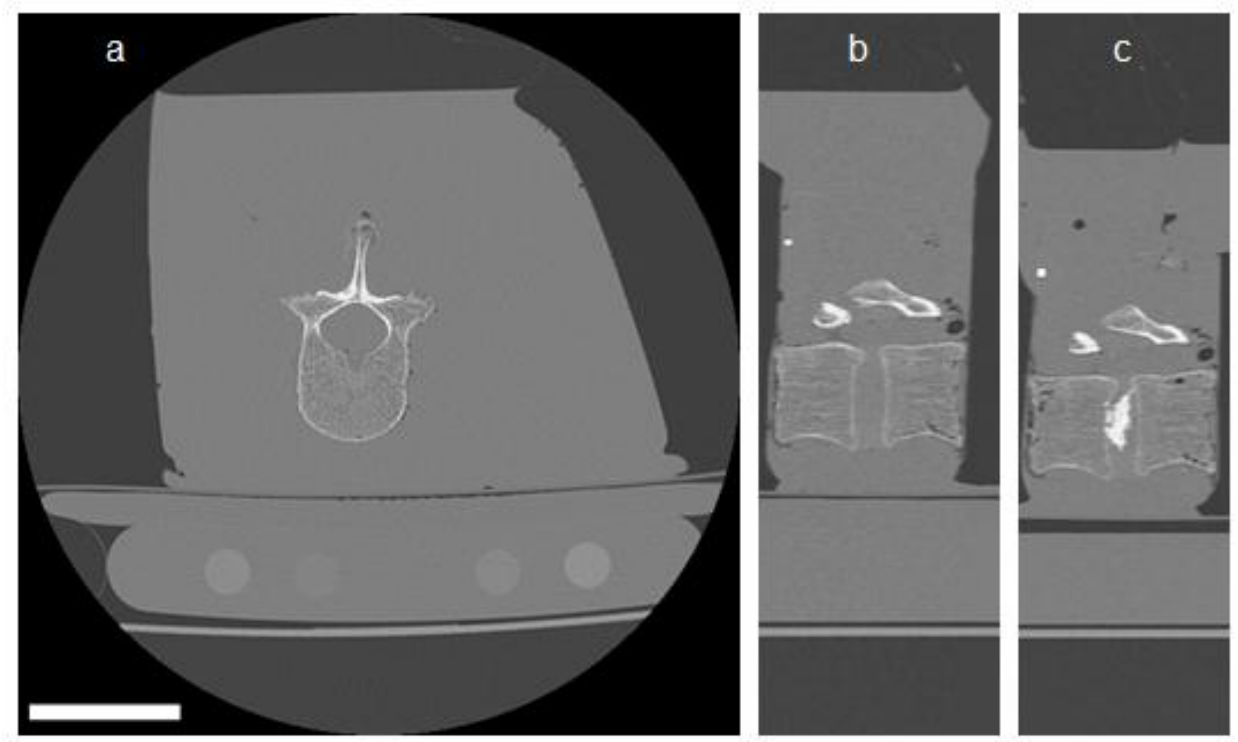

Figure 2. CT scans of a specimen of the cement spacer group. (a) Transverse CT slice showing one vertebral body of the motion segment in the water filled tank and the calibration phantom with five rods of different densities. White scale bar: $50 \mathrm{~mm}$. (b) Mid-sagittal slice prior to operation with the intervertebral disc being intact. Note the glass bead marker of the embedding layer appearing as a bright dot. (c) Post operative mid-sagittal CT slice demonstrating the cement injected into the space of the removed nucleus between the adjacent vertebral bodies.

Destructive mechanical testing of the specimens were then performed by applying uniaxial compressive loading on the outer surfaces of the embedding layers by means of a servohydraulic testing device (Instron 8872, Instron, Norwood, USA). The samples were thawed at room temperature for 4-6 hours prior to testing. Loading rate of the displacement driven experiments was set to $5 \mathrm{~mm} / \mathrm{min}$ to ensure quasi-static conditions, the displacement of the cross-head and the force in the load cell were recorded. The ultimate state was defined either as $20 \%$ reduction in the resisting force or as $20 \%$ total deformation. CT imaging was repeated after the tests with the aim to assess the final damage pattern. The test results of two specimens were not usable and thus these were excluded from further analyses.

The post-operative CT images were segmented by means of a semi-automated approach using the software tool ZibAmira (Zuse Institute Berlin, Germany) and the following compartments were separated: the cortical and trabecular bone volumes of both vertebral bodies, the articular cartilage layers at the joints of the vertebral posterior elements, the remnants of the annulus of the intervertebral disc as well as the PEEK or the bone cement spacer, respectively. The embedding layers were added as rectangular regions thickness and orientation of which were adjusted using the glass bead positions. Case-specific finite element meshes of each motion segment were then generated based on these images, all domains were meshed with linear tetrahedral elements using the CGAL library (www.cgal.org). Maximal cell size was set to $1.4 \mathrm{~mm}$ and the size of the tetrahedrons was adjusted to the local dimensions of the geometrical features (Figure $3 a$ ). 
a

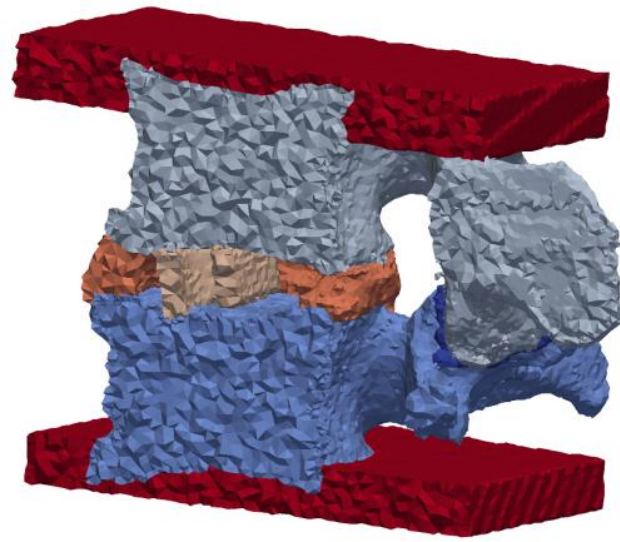

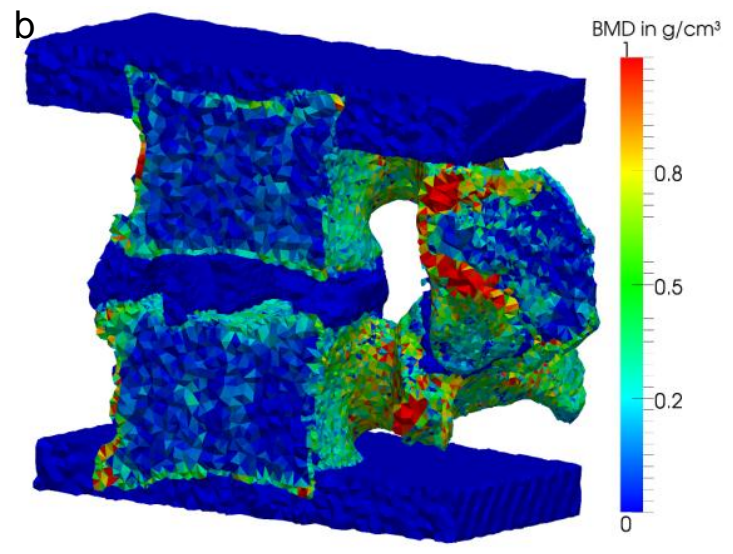

Figure 3. Finite element model of a motion segment stabilized with a PEEK spacer. Half of the model cropped by the mid-sagittal plane is made invisible to show the inner details. (a) The tetrahedral mesh with the different compartments (inferior vertebra, superior vertebra, articular cartilage, disc annulus, PEEK spacer, top and bottom embedding layers) distinguished by colors. (b) Average BMD in the bone elements as computed from the underlying QCT images. The non-bony parts are shown with 0 value (dark blue).

Material properties of the soft tissues, spacer, cement and embedding were assumed to be isotropic, homogeneous and linear elastic. For the two latter materials the elastic modulus was measured experimentally on small cylindrical samples. The properties of PEEK, articular cartilage and annulus were taken from literature. Bone was assumed to be a transversely isotropic, inhomogeneous and linear elasto-plastic material. For both the trabecular and cortical bone compartments the properties of the elements were scaled based on the CT images. Towards this end, the Hounsfield (HU) values were first converted into bone mineral density (BMD) units using the conversion rule assessed as the linear regression between the computed volumetric average $\mathrm{HU}$ value and the known mean mineral density of the five distinct rods of the calibration phantom appearing on each CT image (Figure 2a). The mean BMD value of each element was then determined using a custom written Matlab script (Matlab2009b, Mathworks Inc., Natick, USA) by identifying the image voxels centers of which fell within the element boundaries and taking the average density value of these (Figure 3b). The elements were categorized into 255 sets based on their mean densities. For each set, the elastic moduli and the yield strength were computed using experimentally established vertebra-specific relationships. ${ }^{8-9}$ The applied material properties are summarized in Table 1.

\begin{tabular}{lcccc}
\hline \multicolumn{1}{c}{ Material } & Type & Anisotropy & Young's mod. in MPa & Poisson's ratio \\
\hline Spacer (PEEK) & Linear elastic & Isotropic & 4000 & 0.4 \\
Bone cement & Linear elastic & Isotropic & 10000 & 0.3 \\
Embedding plastic & Linear elastic & Isotropic & 700 & 0.3 \\
Articular cartilage & Linear elastic & Isotropic & 5 & 0.45 \\
Disc annulus & Linear elastic & Isotropic & 10 & 0.45 \\
Bone & Linear elasto- & Transversely & BMD-based & \\
& plastic & isotropic & & 0.381 and 0.104 \\
\hline
\end{tabular}

Table 1. Material properties used in the finite element models. 
Boundary conditions were set to represent the mechanical tests. The lower plane of the inferior embedding layer as fully constrained. To approximate the loading, two distinct type of boundary conditions were used. In the first, more simplified case the uniaxial compressive displacement load was applied directly on the top surface of the upper embedding and a simple ramp scheme was used. In the second one, in order to better mimic the mechanical testing setup, an additional rectangular volume, parallel with the inferior embedding layer and representing the loading plate of the testing device, was included into the model and positioned above the superior embedding. Contact was defined between the upper surface of the superior embedding and the lower plane of the loading plate and the displacement load was applied on the upper surface of the loading plane along the direction of the plane normal. The embedding layers, bone cement and spacer were assumed to be rigidly bound to the bone surfaces.

The facet joints of the vertebrae were only approximately included into the models as the applied imaging modality could not capture the full level of details. In particular, the boundary and thickness of the articular cartilage layers could not be properly assessed. This joint was therefore simplified in the model, and as a simplified solution the cartilage layer was assumed to be very soft. A more proper approximation was targeted in a second approach, where cartilage layer was added only to one articular surface by defining a minimal constant gap between the outer surface of this cartilage layer and the articular bone surface and frictionless contact was defined between these both surfaces.

The nonlinear finite element simulations were performed in Abaqus v6.10 (SIMULIA, Dassault Systemes, Velizy-Villacoublay, France) and ANSYS v14.0 (ANSYS Inc., Southpointe, USA).

\section{Results}

The results of the experimental tests are shown in details in the paper "Biomechanical evaluation of interbody devices by using mechanical compressive test: PEEK spacers versus PMMA cement osteoplasty" published in the same Proceedings Book. The processing of all simulation data is currently in progress and therefore we cannot present the complete set of results. Instead, we show and discuss the first outcomes of the finite element analyses.

The case-specific models allowed detailed evaluation of the initial position, orientation and contact conditions of the cement and PEEK spacers, which showed considerable variations among the specimens of both groups. The contact surface between the cement and the vertebral endplates were quantified based on the meshes and was found to be $536.0 \pm 82.9 \mathrm{~mm}^{2}$ on average in three samples.

The force-displacement curves on the virtual mechanical tests were assessed by taking the summed nodal reaction forces extracted from the constrained surface of the inferior embedding layer and plotting this versus the applied displacement. Figure $4 a$ shows the comparison of two specimens fixed with PEEK and cement spacers, respectively. The motion segment with cement spacer exhibits a larger initial stiffness, but a similar ultimate force as the one with the conventional spacer. The cement case have a more distinct yield point, while the PEEK spacer seems to cause a more continuously softening. All these are in line with the experimental 
observations. A more through comparison of the simulation results with the experimental ones is in progress.

The effect of the boundary conditions is demonstrated in Figure 4b. The force-displacement curve of the models loaded through the stiff top plate exhibit an initial toe region, which resembles better the experimental results and is due to fact that during the first part of the loading the superior embedding progressively becomes more parallel with the inferior one. This is followed by a stiff part which corresponds to the shifted curve of the more simple loading situation. The differences between the two approaches to model the facet joint is in progress, the first results show that these do not have significant effect in the initial part of the loading process.

a

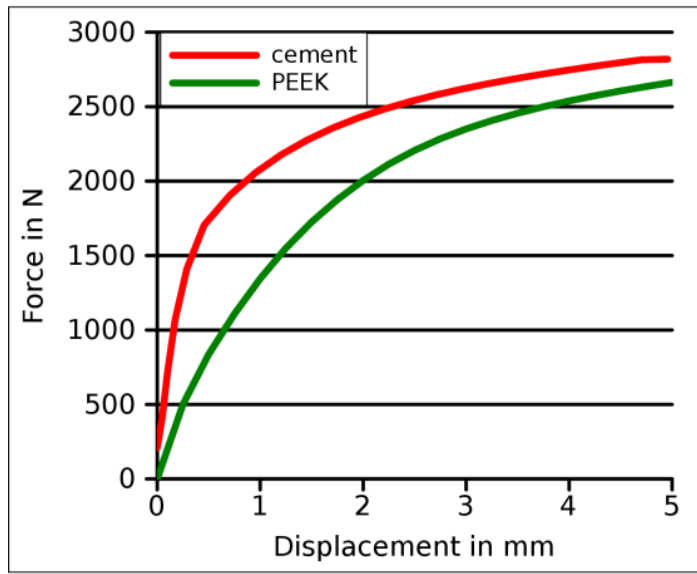

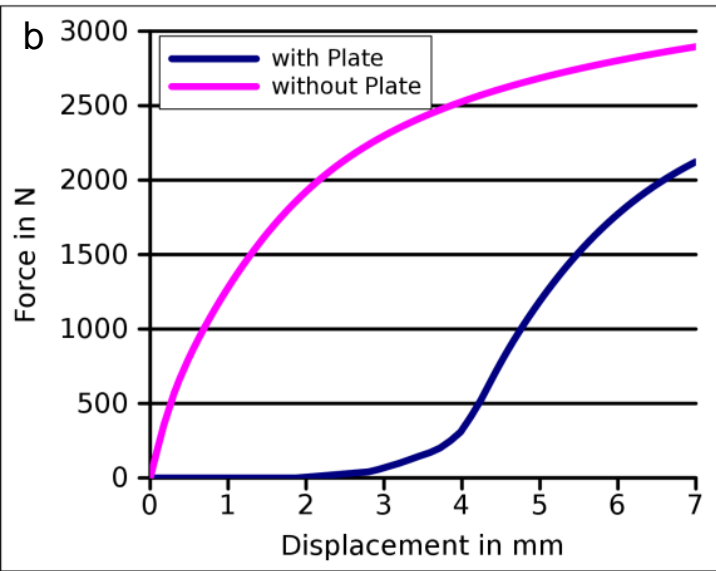

Figure 4. Force-displacement curves obtained from the finite element simulations. (a) Results of motion segments fixed with the cement and the PEEK spacer fixation. (b) Results of a motion segment fixed with

PEEK spacer, loaded with either an additional plate (with plate) or directly through the embedding (without plate).

Contour plots of the plastic strains at each analysis step allowed to evaluate the initial location and evolution of damage zones within the bone compartments in coarse of the loading. The plastic deformation is more homogeneously distributed over a larger volume within the vertebral bodies (Figure 5 a), while the conventional spacer, contacting the endplates on a relatively small area, causes a localized damage in both endplate and trabecular bone (Figure 5b).
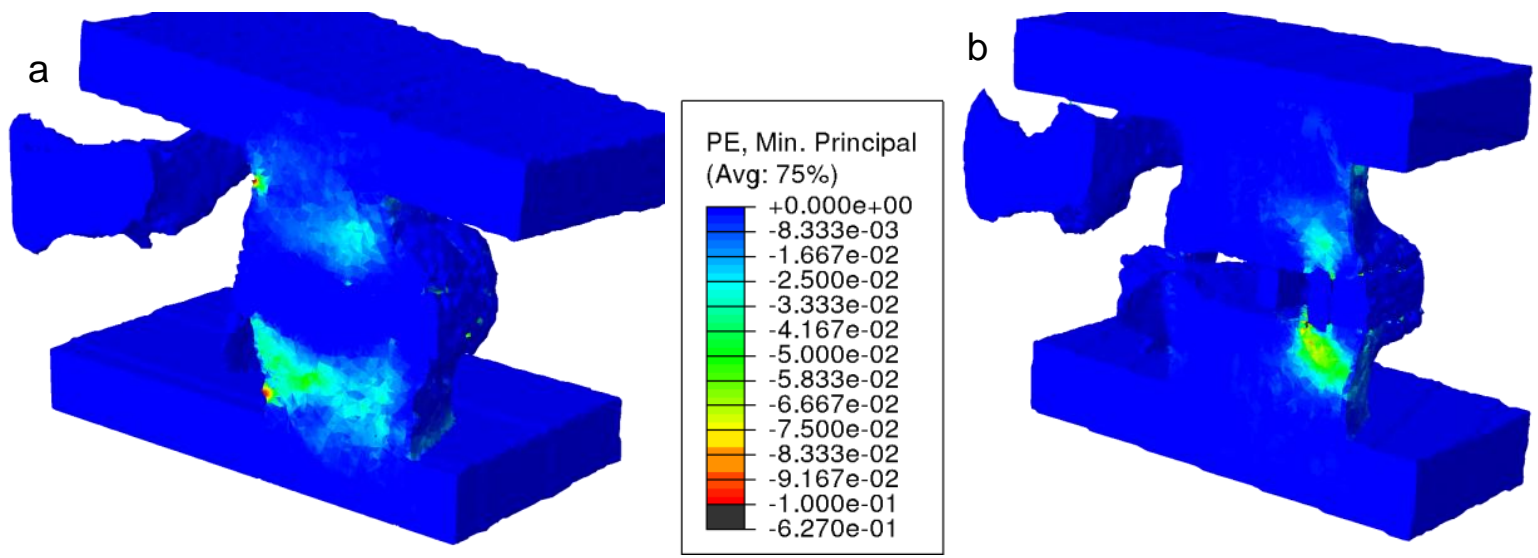

Figure 5. Plastic strains in the initial loading phase shown in a mid-sagittal cut plane of the finite element models of motion segments fixed with cement (a) and conventional spacer (b). 


\section{Discussion}

This paper presents the first results of a finite element simulation approach targeting the evaluation and comparison of the biomechanical properties of human vertebral motion segments fixed with two different interbody devices. Validation of the models with the quantitative results of the mechanical tests and the qualitative results of the post-experimental CT images is currently in progress.

The models seem to properly capture the softer behavior of the PEEK spacer case and the similar ultimate force of the both interbody devices. However, loading of the cement model produces a more distinct yield point, while the PEEK spacer appears to cause a more continuously softening, which is not fully in line with the experimental observations. The lack of the initial linear elastic response of the PEEK spacer models may be due to the inaccurate representation of the endplate, which may partially be due to the limited resolution of the QCT and the element sizes used to mesh this domain. Further, this may be attributed to the limitations of the applied material properties. All these details will have to be investigated with special care.

Despite these potential limitations, the preliminary plastic strain plots results reveal that, as expected, the cement spacer, offering a larger contact surface, efficiently reduces the stress concentrations at the bone-cement interface compared to the conventional spacer and thus leads to a more homogeneous distribution of plastic deformations. In fact, the conventional spacer seems to first perforate the endplate locally by shearing and the further loading involves only a rather small trabecular volume (Figure 5 a). In turn, the larger cement bulk deforms the endplate as a whole in bending mode and therefore the plastic deformation accumulates first in the trabecular compartment rather than the endplate (Figure 5b). The endplate is thus subjected to smaller local stresses and a larger trabecular volume is involved into the resistance. These may be the reason for the higher initial stiffness of the cement spacer cases compared to the conventional ones, which exhibit an early reduction of stiffness. These differences can be crucial from a clinical point of view as the cement spacers, offering higher initial stiffness, may more efficiently inhibit excessive spacer subsidence and further complications. These early findings must be supported by the analysis of further specimens, however, are in line with the experimental results. Further, these results may help to understand the chronology of the damage processes, i.e. at which location the plastic zone was initiated and how it was propagating. Both models shown in Figure 5 suggest the major damage to take place in the inferior vertebra. The important, but complex information carried by the spatial maps of plastic deformations will be compared at the ultimate state with the fracture patters extracted from the post-experimental CT scans.

The available results allow to conclude also that the embedding has a significant effect in the mechanical behaviour of the segments. In particular, the plastic deformation seems to localize also at the cortex-embedding interface (Figure 5 a). These boundary conditions were selected for the sake of the experimental tests and differ considerably from the physiological conditions and do not appropriately represent the in vivo case. Having the QCT-based case-specific models at hand will allow us to investigate the influence of selected parameters on the outcomes in particular, the effect of the embedding layers. Preliminary results indicate that removal of these layers alters the results significantly, non-embedded segments are softer and weaker. Moreover, the intact models can be generated from the CT images assessed prior to operation and the 
differences in transmission of forces between the quasi-native anatomy and the post operative situation can be evaluated.

The models generated with the presented approach are suitable for the purposes of optimization aiming at defining the ideal positioning of the conventional spacers as well as the optimal location, volume and material properties of the cement spacers.

\section{Conclusion}

The finite element simulations of human vertebral motion segments fixed with two distinct interbody devices efficiently complement the mechanical tests and allow detailed investigation and better understanding of the experimental outcomes. Further, these models can be used to analyze and optimize the effect of different parameters like spacer location and material properties, which may help to improve the effectiveness and success of the surgical interventions and ultimately to reduce the occurrence of potential complications

\section{REFERENCES}

1. Jost B, Cripton PA, Lund T, Oxland TR, Lippuner K, Jaeger Ph, Nolte LP. Compressive strength of interbody cages in the lumbar spine: the effect of cage shape, posterior instrumentation and bone density. Eur Spine J 1998; 7(2):132-41.

2. Lim TH, Kwon H, Jeon $\mathrm{CH}, \mathrm{XXXX}$ Effect of endplate conditions and bone mineral density on the compressive strength of the graft-endplate interface in anterior cervical spine fusion. Spine 2001; 26(8):951-6.

3. Tan JS, Bailey CS, Dvorak MF, Fisher CG, Oxland TR. Interbody Device Shape and size are important to strengthen the vertebra-implant interface, Spine 2005; 30(6):638-44.

4. Oxland T, Lund T, Jost B, Cripton P, Lippuner K, Jaeger Ph, Nolte LP.. The relative importance of vertebral bone density and disc degeneration in spinal flexibility and interbody implant performance. Spine 1996;21:2558-69.

5. Csakany T, Varga PP. Discoplasty in Cases of Degenerative Spine with Complications of Osteoporosis, 52nd Congress of the Hungarian Orthopaedic Society and Section Meeting of the Hungarian Society of Arthroscopy, Szolnok, Hungary, 2009 June 25-27.

6. Csákány T, Rónai M, György ZM, Varga PP. Bone Cement as Intervertebral Spacer in Thoracolumbar Stabilization of Aging Spine - Radiological and Clinical Results, Global Spine Congress, Barcelona, Spain, 2011 March 23-26.

7. Polikeit A, Ferguson JS, Nolte LP, Orr TE. Factors influencing stresses in the lumbar spine after the insertion of intervertebral cages: finite element analysis. Eur Spine J 2003;12:413-20.

8. Kopperdahl DL, Morgan EF, Keaveny TM. Quantitative computed tomography estimates of the mechanical properties of human vertebral trabecular bone. J Orthop Res 2002;20:801-5.

9. Mirzaei M, Zeinali A, Razmjoo A, Nazemi M. On prediction of the strength levels and failure patterns of human vertebrae using quantitative computed tomography (QCT)-based finite element method. J Biomech 2009;42(11):1584-91.

The authors gratefully acknowledge the Hungarian Scientific Research Found (Országos Tudományos Kutatói Alapprogramok, OTKA) for providing financial support in frame of 
the grant K-075018. We thank Stefan Fröhlich for his contribution in the finite element modeling process. 